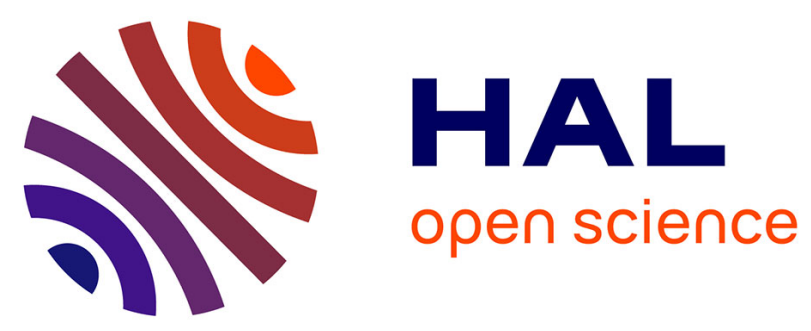

\title{
Caring about the human operator: haptic shared control for enhanced user comfort in robotic telemanipulation
}

\author{
Rahaf Rahal, Giulia Matarese, Marco Gabiccini, Alessio Artoni, Domenico
}

Prattichizzo, Paolo Robuffo Giordano, Claudio Pacchierotti

\section{- To cite this version:}

Rahaf Rahal, Giulia Matarese, Marco Gabiccini, Alessio Artoni, Domenico Prattichizzo, et al.. Caring about the human operator: haptic shared control for enhanced user comfort in robotic telemanipulation. IEEE Transactions on Haptics (ToH), 2020, 13 (1), pp.197-203. 10.1109/TOH.2020.2969662 . hal-02459168

\section{HAL Id: hal-02459168 \\ https://hal.inria.fr/hal-02459168}

Submitted on 29 Jan 2020

HAL is a multi-disciplinary open access archive for the deposit and dissemination of scientific research documents, whether they are published or not. The documents may come from teaching and research institutions in France or abroad, or from public or private research centers.
L'archive ouverte pluridisciplinaire HAL, est destinée au dépôt et à la diffusion de documents scientifiques de niveau recherche, publiés ou non, émanant des établissements d'enseignement et de recherche français ou étrangers, des laboratoires publics ou privés. 


\title{
Caring about the human operator: haptic shared control for enhanced user comfort in robotic telemanipulation
}

\author{
Rahaf Rahal, Giulia Matarese, Marco Gabiccini, Alessio Artoni, \\ Domenico Prattichizzo, Paolo Robuffo Giordano, Claudio Pacchierotti
}

\begin{abstract}
Haptic shared control enables a human operator and an autonomous controller to share the control of a robotic system using haptic active constraints. It has been used in robotic teleoperation for different purposes, such as navigating along paths minimizing the torques requested to the manipulator or avoiding possibly dangerous areas of the workspace. However, few works have focused on using these ideas to account for the user's comfort. In this work, we present an innovative haptic-enabled shared control approach aimed at minimizing the user's workload during a teleoperated manipulation task. Using an inverse kinematic model of the human arm and the Rapid Upper Limb Assessment (RULA) metric, the proposed approach estimates the current user's comfort online. From this measure and an a priori knowledge of the task, we then generate dynamic active constraints guiding the users towards a successful completion of the task, along directions that improve their posture and increase their comfort. Studies with human subjects show the effectiveness of the proposed approach, yielding a 30\% perceived reduction of the workload with respect to using standard guided humanin-the-loop teleoperation.
\end{abstract}

Index Terms-Shared Control, Ergonomics

\section{INTRODUCTION}

Shared control algorithms enable to share the available degrees of freedom of the robotic system between the operator and an autonomous controller. Applications include mobile robotics [1], robot-assisted surgery [2], and assistive robotics [3], [4]. Possible implementations of this approach can be obtained using variable admittance control [5], virtual non-holonomic constraints [6]-[8], and online adaptation of the level of support [9]. Abbink et al. [10] reviewed several implementations of haptic shared control, arguing that it can be useful to meet common design guidelines for the interaction between humans and autonomous controllers. More recently, Hong and Rozenblit [11] presented a haptic shared control approach for surgical training. The trainee is guided away from dangerous areas of the environment using active constraints whose magnitudes change according to the trainee's proficiency level. Similarly, Ghalamzan et al. [12] presented a haptic shared control for teleoperated grasping. The operators are in full control of the robotic manipulator and capable of choosing any grasping pose they like. At the same time, an active constraint guides them toward the direction that optimizes the end-effector manipulability over the post-grasp trajectory.

Haptic shared control has been successfully used to perform different tasks, e.g., to guide the operator toward a reference position [13][17], to avoid certain areas of the environment [15], [18]-[20], and for learning manual tasks [21], [22]. However, researchers have rarely

R. Rahal and G. Matarese are with Univ Rennes, Inria, CNRS, IRISA, Rennes, France, \{rahaf.rahal, giulia.matarese\}@irisa.fr. These two authors contributed equally to the manuscript.

M. Gabiccini and A. Artoni are with the Dip. Ingegneria Civile e Industriale, Univ. Pisa, Pisa, Italy, \{m.gabiccini,a.artoni\}@ ing.unipi.it. M. Gabiccini is also with the Centro di Ricerca "E. Piaggio", Univ. Pisa, Pisa, Italy.

D. Prattichizzo is with the Dip. Ingegneria dell'Informazione e Scienze Matematiche, Univ. Siena, Siena, Italy and with the Dept. Advanced Robotics, Istituto Italiano di Tecnologia, Genova, Italy, prattichizzo@diism.unisi.it.

P. Robuffo Giordano, and C. Pacchierotti are with CNRS, Univ Rennes, Inria, IRISA, Rennes, France, \{prg, claudio.pacchierotti\}@irisa.fr.

The authors want to thank Charles Pontonnier for his help in defining the human effort cost function.

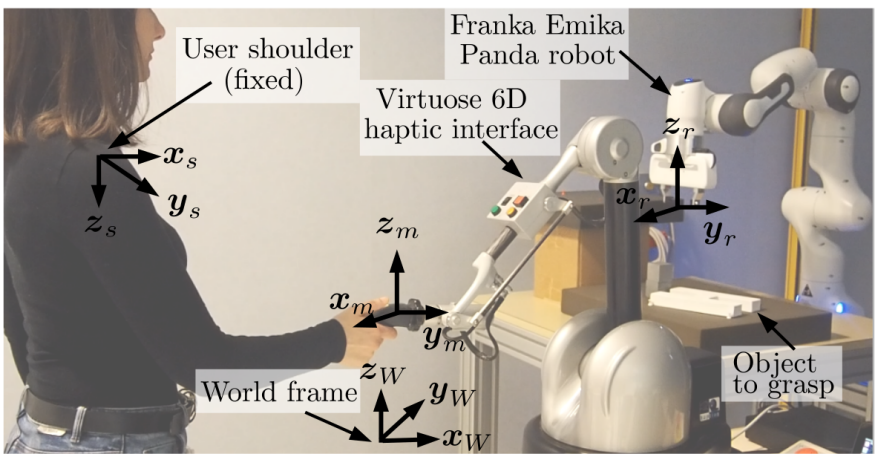

Fig. 1. The experimental setup for the pick and place task. On the master side, a Haption Virtuose 6-DoF haptic device, on the slave side a 7-DoF Franka Emika Panda robot. The user shoulder is assumed to be fixed.

focused on the user's comfort during robotic telemanipulation, and never - to the best of our knowledge - has haptic shared control been designed for this specific purpose. Nonetheless, this is a very important issue, as operators in many high-impact scenarios use the robotic teleoperation system for long uninterrupted time periods (e.g., a robotic prostatectomy generally takes $2-4$ hours). To our knowledge, a few examples are available in the field of human robot interaction (HRI). For example, Busch et al. [23] used a simplified human model to calculate the user's body configuration in HRI. They derived a continuous cost function based on the Rapid Entire Body Assessment score (REBA) and used it to choose the robot position optimizing the human joint angles (and thus the ergonomic comfort). Marin et al. [24] optimized the ergonomics of an HRI task where subjects are asked to drill on a board carried by the robot. They relied on musculoskeletal simulations to train a Contextual Ergonomics Model through a probabilistic supervised learning Gaussian process. Chen et al. [25] modeled the human arm as a 7-degrees-of-freedom (7-DoF) robotic manipulator, and its muscular effort was derived based on the estimated value of the joint torques. Similarly, Peternel et al. [26] estimated muscular effort from torques during co-manipulation tasks.

This paper introduces a haptic shared control technique minimizing the user's workload during robotic teleoperation. Using an inverse kinematic model of the human arm and an online implementation of the Rapid Upper Limb Assessment (RULA) tool [27], the proposed approach starts by estimating the current user's discomfort at runtime. Then, this metric is combined with some knowledge of the target task and system (e.g., direction to follow, target position to reach, effort demanded to the robot) to generate dynamic active constraints guiding the user towards a successful completion of the task along directions that require a reduced workload. The user is nevertheless always in control of the robot motion through the master interface and can deviate from the proposed path if needed, similarly to [12]. This approach is compatible with any robotic teleoperation framework and can be combined with as many additional pieces of information on the task and system as needed. To demonstrate the validity of the proposed approach, we carried out a bilateral telemanipulation experiment with 15 participants, evaluating the effect of our approach 
in the task's performance and in the workload perceived by the users.

\section{SYSTEM DETAILS}

\section{A. System model}

The master side is composed of a torque-controlled Haption Virtuose 6-DoF haptic interface, while the slave side consists of a Franka Panda 7-DoF manipulator equipped with a gripper. Fig. 2 gives an overview of the entire system architecture.

We consider four reference frames in our system (see Fig. 1): $\mathcal{F}_{r}:\left\{\mathcal{O}_{r}, \boldsymbol{x}_{r}, \boldsymbol{y}_{r}, \boldsymbol{z}_{r}\right\}$ is the robot frame attached to the gripper; $\mathcal{F}_{m}:\left\{\mathcal{O}_{m}, \boldsymbol{x}_{m}, \boldsymbol{y}_{m}, \boldsymbol{z}_{m}\right\}$ is the frame attached to the end-effector of the master interface; $\mathcal{F}_{s}:\left\{\mathcal{O}_{s}, \boldsymbol{x}_{s}, \boldsymbol{y}_{s}, \boldsymbol{z}_{s}\right\}$ is the frame attached to the shoulder of the user, which is assumed to be fixed; and $\mathcal{F}_{W}:\left\{\mathcal{O}_{W}, \boldsymbol{x}_{W}, \boldsymbol{y}_{W}, \boldsymbol{z}_{W}\right\}$ is the world frame. Let $\mathbf{x}_{m}=$ $\left(\mathbf{p}_{m}, \mathbf{R}_{m}\right) \in \mathbb{R}^{3} \times \mathcal{S O}(3)$ be the pose of the master interface endeffector, $\mathbf{x}_{r}=\left(\mathbf{p}_{r}, \mathbf{R}_{r}\right) \in \mathbb{R}^{3} \times \mathcal{S O}(3)$ the pose of the robot end-effector, and $\mathbf{x}_{s}=\left(\mathbf{p}_{s}, \mathbf{R}_{s}\right) \in \mathbb{R}^{3} \times \mathcal{S O}(3)$ the pose of the user's shoulder, all expressed in the common world frame $\mathcal{F}_{W}$. The linear/angular velocities of the master and slave in $\mathcal{F}_{W}$ are denoted by $\boldsymbol{v}_{m}=\left(\dot{\boldsymbol{p}}_{m}^{T}, \boldsymbol{\omega}_{m}^{T}\right)^{T} \in \mathbb{R}^{6}$ and $\boldsymbol{v}_{r}=\left(\dot{\boldsymbol{p}}_{r}^{T}, \boldsymbol{\omega}_{r}^{T}\right)^{T} \in \mathbb{R}^{6}$, respectively.

The master device is modeled as a generic, gravity precompensated, mechanical system

$$
\boldsymbol{M}_{m}\left(\mathbf{x}_{m}\right) \dot{\boldsymbol{v}}_{m}+\boldsymbol{C}_{m}\left(\mathbf{x}_{m}, \boldsymbol{v}_{m}\right) \boldsymbol{v}_{m}=\boldsymbol{f}_{m}+\boldsymbol{f}_{h},
$$

where $\boldsymbol{M}\left(\mathbf{x}_{m}\right) \in \mathbb{R}^{6 \times 6}$ is the positive-definite and symmetric inertia matrix, $\boldsymbol{C}\left(\mathbf{x}_{m}, \boldsymbol{v}_{m}\right) \in \mathbb{R}^{6 \times 6}$ represents the Coriolis/centrifugal terms, $\boldsymbol{f}_{h} \in \mathbb{R}^{6}$ is the spatial force applied by the human operator to the master interface, and $\boldsymbol{f}_{m} \in \mathbb{R}^{6}$ the feedback forces provided to the operator. On the other hand, the slave robot is controlled in velocity,

$$
\boldsymbol{v}_{r}=\boldsymbol{v}_{m}+\lambda\left[\begin{array}{c}
\mathbf{p}_{r, d}-\mathbf{p}_{r} \\
\mathbf{R}_{r}^{r}(\theta \mathbf{u})_{r, d}
\end{array}\right],
$$

where $\lambda$ is a gain parameter, $\mathbf{p}_{r, d}=\left(\mathbf{p}_{m}-\mathbf{p}_{m 0}\right)+\mathbf{p}_{r 0}$ is the desired robot position calculated from the current master position $\mathbf{p}_{m}$, and $\mathbf{p}_{m 0}$ and $\mathbf{p}_{r 0}$ are the initial poses of the master and the slave. For the angular velocity term, we rely instead on ${ }^{r}(\theta \boldsymbol{u})_{r, d}$, the angleaxis representation of the relative rotation between the desired and the current slave orientations [28].

\section{B. Human arm model}

To estimate the user's discomfort due to arm posture in real-time, we need an estimate of the configuration of his/her arm throughout the teleoperation task. In fact, in this work, we decided to rely on a non-invasive technique for estimating the ergonomic comfort, using only the joint angles of the arm. We will consider other approaches in the future (see Sec. V).

For this purpose, the human arm is modeled as a 7-DoF robotic arm, similarly to the work of Shimizu et al. [29]. A spherical joint is used for representing the shoulder and the wrist, while the elbow is represented by a revolute joint (Fig. 3a). We use an XYZ Euler convention to represent the spherical joints, to be consistent with the RULA metric which will be introduced in Sec. II-C. Given that the operator is asked to keep the shoulder at a fixed position throughout the task, we can estimate the joint values of the user's arm $\boldsymbol{q}_{\text {arm }}=\left[q_{1}, \ldots, q_{7}\right]^{T}$ using solely the position of the master end effector, which coincides with the user's palm, using inverse kinematics (details in Sec. II-D).

\section{User workload parametrization}

As mentioned in Sec. I, human posture is often used to estimate and prevent work-related musculoskeletal risks, as posture is indeed one of the risk factors for workers. In this respect, the Rapid Upper

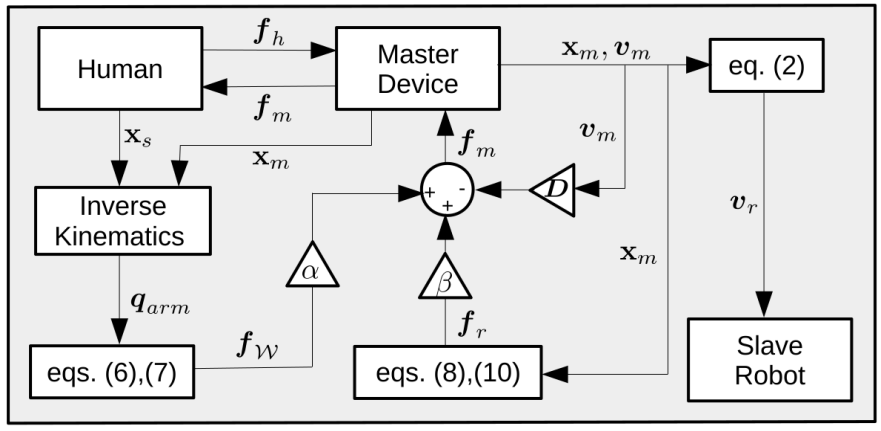

Fig. 2. Block diagram summarizing the system architecture and the shared control algorithm presented in Secs. II and III.

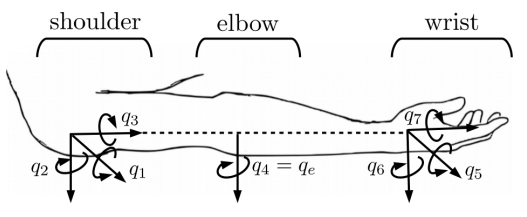

(a)

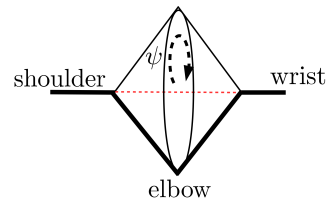

(b)
Fig. 3. (a) The joints for the human arm. (b) Definition of the arm angle $\psi$.

Limb Assessment (RULA) method [27] is a discrete metric in which a score is given for each upper limb configuration. The sum of these scores represents the overall workload experienced by the user.

In this paper, the load or discomfort estimate needs to be continuously updated as a function of the user arm configuration throughout the teleoperation task. To do so, we derive a RULA-inspired continuous metric, which increases as the deviation from the resting and most comfortable position of each joint angle in the arm increases. We calculate, at each time step, $\boldsymbol{q}_{s}=\left[q_{1}, q_{2}, q_{3}\right]^{T}$ the shoulder angles, $q_{e}=q_{4}$ the elbow angle, and $\boldsymbol{q}_{w}=\left[q_{5}, q_{6}, q_{7}\right]^{T}$ the wrist angles. As such, $\boldsymbol{q}_{\mathrm{arm}}=\left[\boldsymbol{q}_{s}^{T}, q_{e}, \boldsymbol{q}_{w}^{T}\right]^{T}$ (see Fig. 3a). The workload $\mathcal{W}$ is then defined as the sum of the squared differences between the angles and their rest positions $(\pi / 2$ for the elbow angle, and 0 for the others)

$$
\mathcal{W}=\boldsymbol{q}_{s}^{T} \boldsymbol{q}_{s}+\left(q_{e}-\pi / 2\right)^{2}+\boldsymbol{q}_{w}^{T} \boldsymbol{q}_{w} .
$$

\section{Inverse kinematics and solving the redundancy}

Calculating the workload $\mathcal{W}$ assumes that the joint angles $\boldsymbol{q}_{\text {arm }}$ of the user arm are known at each time step. However, given that the arm is represented with a 7-DoF kinematic model, the inverse kinematics is not straightforward. Similarly to [30], we define an arm angle $\psi \in[0, \pi]$, which represents the swivel angle of the arm around a virtual line connecting the shoulder to the wrist, as shown in Fig. 3b. If $\psi$ is known, the redundancy is resolved, since the shoulder and wrist angles can be parametrized using it, and the elbow angle $q_{e}$ can be computed solely based on geometry.

At each time step, we calculate the inverse kinematics result $\boldsymbol{q}_{\text {arm }}$ from the user hand position, using all possible values of $\psi$ (discrete values with increments of 0.01 in the $[0, \pi]$ range). Then, we compute the corresponding $\mathcal{W}$ value assuming that the user naturally chooses the most comfortable configuration of the elbow when presented with a set of options. To do so, we choose the inverse kinematics solution corresponding to the $\psi$ value leading to the least discomfort between the possible solutions (lowest $\mathcal{W}$ ), similarly to what was done in [25]. This assumption only affects the swivel angle of the elbow and no other joint angle. Additional techniques that we will use in the future to improve this pose estimation are mentioned in Sec. V. Finally, a condition is added to ensure that the resulting angles are close to the previous configuration of the arm, so that no abrupt changes occur. 


\section{SHARED CONTROL ARCHITECTURE}

This Section describes the shared control algorithm used to guide the human operator during the teleoperation task. The main goal of this architecture is not only to account for task-related requirements, but to also target the maximization of the user's comfort during the task. We thus divide our haptic feedback force into two components: a human-related component, $\boldsymbol{f}_{\mathcal{W}}$, and a robot/task-related one, $\boldsymbol{f}_{r}$.

\section{A. Human workload cost function}

We want to minimize the muscular discomfort experienced during the task execution. This is obtained by minimizing the cost function $\mathcal{H}_{\mathcal{W}}=\mathcal{W}$, i.e., the workload. A comfortable arm configuration is thus defined as one with a small value for the workload $\operatorname{cost} \mathcal{W}$.

\section{B. Task-related cost function}

The second component of the haptic feedback is related to the task itself, as in more traditional shared control architectures. A second cost function $\mathcal{H}_{r}$ is thus introduced to represent this task-related metric to be optimized. The cost $\mathcal{H}_{r}$ can be related to maximizing, e.g., the distance from joint limits, singularities, or obstacles. It could also be related to the distance from a target, or to the robot joint velocities or torques, in which case it should be minimized. While this cost function is general, we choose for our application to minimize the Euclidean distance to the target object pose, similarly to [31], by defining

$$
\mathcal{H}_{r}=\left[\begin{array}{c}
\boldsymbol{p}_{r, g}-\boldsymbol{p}_{r} \\
r(\theta \boldsymbol{u})_{r, g}
\end{array}\right]^{T}\left[\begin{array}{c}
\boldsymbol{p}_{r, g}-\boldsymbol{p}_{r} \\
r(\theta \boldsymbol{u})_{r, g}
\end{array}\right],
$$

where $\boldsymbol{p}_{r}$ and $\boldsymbol{p}_{r, g}$ are the current and goal positions of the robot end-effector, and ${ }^{r}(\theta \boldsymbol{u})_{r, g}$ is the angle-axis representation of the relative rotation between the current and target robot poses. The same approach can be easily extended to any similar task-related metric.

\section{Haptic feedback}

We design the haptic feedback to guide the user during teleoperation, such that both task- and human-comfort-inspired cost functions are minimized. To achieve this goal, the forces applied at the master end effector are defined as

$$
\boldsymbol{f}_{m}=\alpha \boldsymbol{f}_{\mathcal{W}}+\beta \boldsymbol{f}_{r}-\boldsymbol{D} \boldsymbol{v}_{m},
$$

where $\boldsymbol{f}_{\mathcal{W}}$ is the force vector instantaneously guiding the user towards the position with the highest comfort, and vector $\boldsymbol{f}_{r}$ is the force minimizing the task cost function, which in our case is related to the distance from the target release position. $\alpha$ and $\beta$ are weights to be tuned depending on the importance to be given to each cost function $(\alpha+\beta=1)$. Finally, $\boldsymbol{D}$ is a diagonal damping matrix to improve the bilateral stability of the system [7], [32]. We chose a damping value of $2 \mathrm{Ns} / \mathrm{m}$ in translation and $0.07 \mathrm{Nms} / \mathrm{rad}$ in rotation.

The feedback component related to $\mathcal{H}_{\mathcal{W}}$ is designed to be proportional to a desired velocity of the master device in a direction that minimizes the cost function,

$$
\boldsymbol{f}_{\mathcal{W}}=K_{\mathcal{W}} \boldsymbol{v}_{m, d_{\mathcal{W}}}
$$

where $K_{\mathcal{W}}$ is a proportional constant, and $\boldsymbol{v}_{m, d_{\mathcal{W}}}$ is the desired velocity of the master device end effector, based on the metric maximizing the comfort. It is calculated from the desired joint velocities of the human arm, $\dot{\boldsymbol{q}}_{\mathrm{arm}, d}$, as follows: $\boldsymbol{v}_{m, d_{\mathcal{W}}}=\boldsymbol{T} \boldsymbol{J} \dot{\boldsymbol{q}}_{\text {arm }, d}$, where $\boldsymbol{T}$ is a transformation matrix to take the desired velocity calculated from the shoulder frame $\mathcal{F}_{s}$ to $\mathcal{F}_{m}$, and $\boldsymbol{J}$ is the Jacobian of the human arm. The velocity $\dot{\boldsymbol{q}}_{\text {arm }, d}$ is chosen for ensuring that $\mathcal{H}_{\mathcal{W}}$ is minimized, or, in other words, $\dot{\mathcal{H}}_{\mathcal{W}}\left(\boldsymbol{q}_{\text {arm }}\right)=\left(\partial \mathcal{H}_{\mathcal{W}} / \partial \boldsymbol{q}_{\text {arm }}\right) \dot{\boldsymbol{q}}_{\text {arm }} \leq 0$. We thus choose the desired angular velocity of the arm angles to be in the negative direction of the gradient of the human-related cost function,

$$
\dot{\boldsymbol{q}}_{\mathrm{arm}, d}=-\frac{\partial \mathcal{H}_{\mathcal{W}}}{\partial \boldsymbol{q}_{\mathrm{arm}}}
$$

Null values for $f_{\mathcal{W}}$ in eq. (6), caused by the algorithm being stuck in a singular configuration $\overline{\boldsymbol{q}}$ (where $\boldsymbol{N}(\boldsymbol{J}(\overline{\boldsymbol{q}})) \neq 0$ ) different from the target one associated with the minimum RULA and where $\dot{\boldsymbol{q}}_{\mathrm{arm}, d} \in$ $\boldsymbol{N}(\boldsymbol{J}(\overline{\boldsymbol{q}}))$, are very unlikely due to the quite limited range of motion of the user's arm.

The feedback component related to $\mathcal{H}_{r}$ is designed similarly,

$$
\boldsymbol{f}_{r}=K_{r} \boldsymbol{v}_{m, d_{r}} .
$$

Since our haptic feedback is applied at the master side, and assuming no significant delays or communication issues occur between the master and the slave, we start by defining a new cost function, $\mathcal{H}_{m}$, which encodes the difference between the master device pose and its target pose, computed from the target robot pose using a transformation matrix:

$$
\mathcal{H}_{m}=\left[\begin{array}{c}
\boldsymbol{p}_{m, g}-\boldsymbol{p}_{m} \\
m(\theta \boldsymbol{u})_{m, g}
\end{array}\right]^{T}\left[\begin{array}{c}
\boldsymbol{p}_{m, g}-\boldsymbol{p}_{m} \\
m(\theta \boldsymbol{u})_{m, g}
\end{array}\right],
$$

where $\boldsymbol{p}_{m}$ and $\boldsymbol{p}_{m, g}$ are the current and target positions of the haptic device end-effector. ${ }^{m}(\theta \boldsymbol{u})_{m, g}$ is the angle-axis representation of the relative rotation between the current and the target master poses. The desired velocity of the haptic device is finally chosen such that it minimizes the distance to the target

$$
\boldsymbol{v}_{m, d_{r}}=\left[\begin{array}{c}
\dot{\boldsymbol{p}}_{m, d_{r}} \\
\boldsymbol{\omega}_{m, d_{r}}
\end{array}\right]=\left[\begin{array}{c}
\boldsymbol{p}_{m, g}-\boldsymbol{p}_{m} \\
m(\theta \boldsymbol{u})_{m, g}
\end{array}\right],
$$

as also done in, e.g., [28].

To avoid having one of the two components of the force $\left(\boldsymbol{f}_{r}\right.$ and $\boldsymbol{f}_{\mathcal{W}}$ ) masking the other one due to their difference in scale, we scale each of the forces to the same range before adding them in eq. (5). The linear and torque components of each spatial force vector are scaled to a norm of $2.5 \mathrm{~N}$ and $0.25 \mathrm{~N} . \mathrm{m}$, respectively. A similar technique was used in [33] to scale the 19 components of a biomimetic sensor so as to be able to combine and compare them. The resulting force guides the users towards the pose minimizing the composite metrics, but it is always gentle enough to enable them to deviate from the suggested path, if needed. Additional feedback techniques for providing this guidance information are discussed in Sec. V. Finally, once the user is within a threshold distance from the target position, we scale each force proportionally to the distance to the goal, to avoid strong forces and oscillations as the user gets closer to the goal (as done in [32]).

\section{EXPERIMENTAL EVALUATION}

\section{A. Setup and Participants}

The experimental setup is shown in Fig. 1 and described in Sec. II. The remote environment is composed of three different sets of objects to grasp, pick up, and place on a target location, as detailed in the next Section. To enable the operator to see the environment, the master interface was placed next to the slave robot.

Fifteen subjects participated in the study (10 males, 5 females). Each subject spent about two minutes practicing the control of the telemanipulation system before starting the experiment. At this moment, the pose of the user's shoulder was measured and kept fixed throughout the experiment. This latter point is important because our workload parametrization and inverse kinematics assume a fixed position of the shoulder (see Secs. II-C and II-D). 


\section{B. Task and Conditions}

Participants used the master interface to control the slave manipulator. The task consisted in grasping the objects (first part of the task) and moving them to a target put-down location marked on the table (second part of the task). Participants were asked to complete the task as precisely and fast as possible. The task started when the manipulator moved for the first time, and it was considered completed when the object was released on the target.

As explained in Sec. III, we combine different cost functions to take into account human-related metrics as well as robot- and taskrelated ones. In this experiment, we consider the estimated human workload as our human-related cost $\mathcal{H}_{\mathcal{W}}$, and the distance from the target location (i.e., the grasping pose in the first part of the task and the put-down location in the second part) as our task-related cost $\mathcal{H}_{r}$. These two functions are then properly weighted and combined to generate the guiding feedback, as described in eq. (5). Of course, the proposed approach can be used for any other set of cost functions.

We consider three different weighting schemes for the contribution of $\mathcal{H}_{\mathcal{W}}$ and $\mathcal{H}_{r}$ to the haptic guidance $\boldsymbol{f}_{m}$ :

(A0) $\alpha=0, \beta=1$ (the human-centered metric is disregarded and the operator is simply guided toward the target);

(A20) $\alpha=0.2, \beta=0.8$ (weak human-centered guidance);

(A40) $\alpha=0.4, \beta=0.6$ (strong human-centered guidance).

We only consider conditions with $\beta>0.5$ to ensure that the guidance feedback always brings the user towards the completion of the task. In fact, a hypothetical human-centered-only condition with $\alpha=1.0, \beta=0$ would simply guide the user towards a comfortable arm position, with no information and guidance regarding the task.

For each weighting condition A0, A20, A40, participants were asked to pick and place three different sets of objects:

(B) an empty cardboard box of dimensions $14 \times 4 \times 4 \mathrm{~cm}$;

(C) two cubes, each of dimensions $4.2 \times 4.2 \times 4.2 \mathrm{~cm}$;

(L) the wooden letter " $\mathrm{H}$ " with outer dimensions $21 \times 13 \times 2.5 \mathrm{~cm}$.

Each subject carried out eighteen randomized repetitions of the pick-and-place task, two for each weighting condition and set of objects. These two repetitions differed in the pick-up and put-down locations. A video is available as supplemental material and at https: //youtu.be/DodGI4wMRFA. Fig. 4 shows the effect of the weighting schemes in a simple reaching movement between two fixed points. Our scenario involved a pick and place task, in which the starting and target positions are placed on two supports having different height. To avoid colliding with any of the supports and make our approach viable for any trajectory, we designed our task-related feedback by introducing an intermediate target point, $\mathbf{x}_{r, i}=\left(\mathbf{p}_{r, i}, \mathbf{R}_{r, i}\right)$, higher than both supports. The user is first guided to this intermediate point. Then, once the robot reaches its neighborhood, the guiding force smoothly switches toward a new target pose, $\mathbf{x}_{r, g}=\left(\mathbf{p}_{r, g}, \mathbf{R}_{r, g}\right)$, which is the final release position for our object. This approach can be easily used with any arbitrarily complex trajectory.

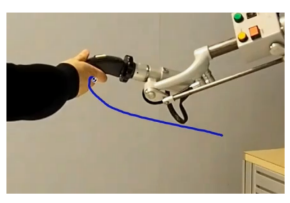

(a) $\mathrm{AO}$

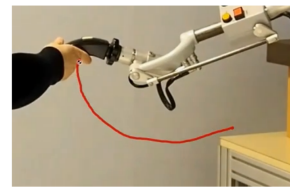

(b) A20

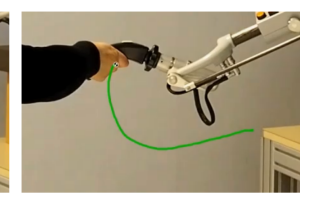

(c) A40
Fig. 4. User trajectory for a reaching movement for the three weightings A0 (blue), A20 (red), and A40 (green). In A0, only $\mathcal{H}_{r}$ is considered, and the user trajectory is therefore almost horizontal. As the contribution of $\mathcal{H}_{\mathcal{W}}$ to the haptic feedback increases, the trajectory tries to minimize the user discomfort by moving the arm to a downward (more comfortable) position.
TABLE I

STATISTICAL ANALYSIS (TWO-WAY REPEATED-MEASURE ANOVA)

\begin{tabular}{|c|c|c|c|}
\hline \multicolumn{4}{|l|}{ Completion time } \\
\hline \multicolumn{4}{|c|}{ Main effect of weighting } \\
\hline A0 vs. A20 & $p=0.020$ & $\mathrm{~A} 0$ vs. $\mathrm{A} 40$ & $p<0.001$ \\
\hline \multicolumn{4}{|c|}{ Main effect of object } \\
\hline B vs. L & $p<0.001$ & L vs. C & $p<0.001$ \\
\hline B vs. C & $p<0.001$ & & \\
\hline \multicolumn{4}{|l|}{ Placing error } \\
\hline \multicolumn{4}{|c|}{ Main effect of object } \\
\hline B vs. C & $p<0.001$ & L vs. C & $p=0.003$ \\
\hline \multicolumn{4}{|l|}{ Average $\mathcal{H}_{W_{-}}$} \\
\hline \multicolumn{4}{|c|}{ Main effect of weighting } \\
\hline A0 vs. A20 & $p=0.048$ & $\mathrm{~A} 0$ vs. $\mathrm{A} 40$ & $p<0.001$ \\
\hline A 20 vs. A40 & $p=0.028$ & & \\
\hline \multicolumn{4}{|c|}{ Main effect of object } \\
\hline B vs. L & $p<0.001$ & L vs. C & $p<0.001$ \\
\hline \multicolumn{4}{|l|}{ Maximum $\mathcal{H}_{\mathcal{W}}$} \\
\hline \multicolumn{4}{|c|}{ Main effect of weighting } \\
\hline A0 vs. A20 & $p=0.049$ & $\mathrm{~A} 0$ vs. $\mathrm{A} 40$ & $p<0.001$ \\
\hline A 20 vs. A40 & $p=0.025$ & & \\
\hline \multicolumn{4}{|c|}{ Main effect of object } \\
\hline B vs. L & $p<0.001$ & L vs. C & $p=0.002$ \\
\hline B vs. C & $p=0.012$ & & \\
\hline \multicolumn{4}{|c|}{ NASA TLX workload index } \\
\hline \multicolumn{4}{|c|}{ Main effect of weighting } \\
\hline A0 vs. A20 & $p=0.035$ & $\mathrm{~A} 0$ vs. $\mathrm{A} 40$ & $p=0.001$ \\
\hline A20 vs. A40 & $p=0.050$ & & \\
\hline
\end{tabular}

\section{Results}

To evaluate the effectiveness of the proposed human-centered shared control approach, we recorded (i) the completion time, (ii) the error in placing the objects at the target, (iii-iv) the mean and maximum $\mathcal{H}_{\mathcal{W}}$ registered, and $(v)$ the NASA Task Load Index (NASA-TLX) [34]. To compare these metrics, we ran two-way repeated-measures ANOVA tests. The three weightings (A0 vs. A20 vs. A40) and the three sets of objects to move (B vs. C vs. L) were treated as within-subject factors. Data were transformed using the arcsin transformation whenever necessary to achieve normality. All data passed the Shapiro-Wilk normality test. A Greenhouse-Geisser correction was used when the assumption of sphericity was violated. Results of post hoc analysis with Bonferroni adjustments are reported in Table I (only significant $p$ values are shown).

Fig. 5a shows the completion time, averaged across trials. All data passed the Mauchly's Test of Sphericity. The two-way repeatedmeasure ANOVA revealed a statistically significant change for this metric across weighting conditions $(\mathrm{F}(2,28)=14.898, p<0.01)$ and objects $(\mathrm{F}(2,28)=107.168, p<0.001)$. Fig. 5b shows the error in placing the objects, averaged across trials. It is calculated as the distance between the target position and where the objects were actually placed at the end of the task. All data passed the Mauchly's Test of Sphericity. The two-way repeated-measure ANOVA revealed a statistically significant change for this metric across objects only $(\mathrm{F}(2,28)=16.118, p<0.001)$. Fig. 5c shows the mean $\mathcal{H}_{\mathcal{W}}$, averaged across trials. It is calculated as the value of $\mathcal{H}_{\mathcal{W}}$ averaged over the duration of the task. All data passed the Mauchly's Test of Sphericity. The two-way repeated-measure ANOVA revealed a statistically significant change for this metric across weighting conditions $(\mathrm{F}(2,28)=17.287, p<0.001)$ and objects $(\mathrm{F}(2.28)=$ 58.534, $p<0.001)$. Fig. 5d shows the maximum $\mathcal{H}_{\mathcal{W}}$, averaged across trials. It is calculated as the maximum value of $\mathcal{H}_{\mathcal{W}}$ registered during the task. All data passed the Mauchly's Test of Sphericity. The two-way repeated-measure ANOVA revealed a statistically significant change for this metric across weighting conditions $(\mathrm{F}(2,28)=16.594$, $p<0.001)$ and objects $(\mathrm{F}(2.28)=32.418, p<0.001)$. Fig. $5 \mathrm{e}$ shows the overall workload score of the NASA TLX, registered 


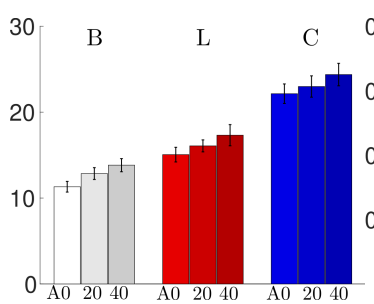

(a) Completion time (s).

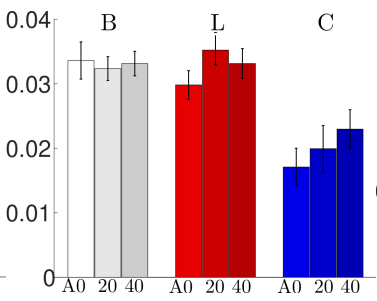

(b) Error placing the objects $(\mathrm{m})$.

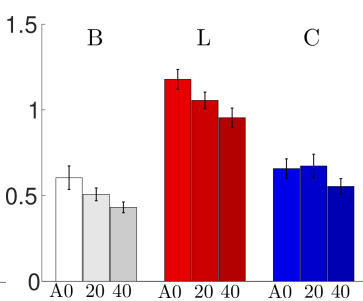

(c) Average $\mathcal{H}_{\mathcal{W}}$ value $\left(\operatorname{rad}^{2}\right)$.

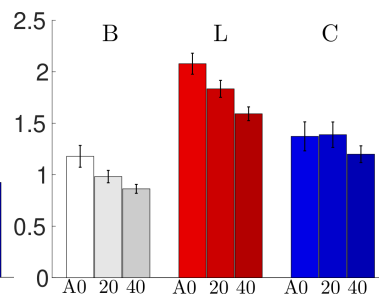

(d) Maximum $\mathcal{H}_{\mathcal{W}}$ value $\left(\operatorname{rad}^{2}\right)$.

Fig. 5. Human subjects experiment. Mean and standard error of the mean of (a) completion time, (b) error in placing the objects, (c) average $\mathcal{H}_{\mathcal{W}}$, (d) maximum $\mathcal{H}_{\mathcal{W}}$, and (e) NASA TLX load index for the three control conditions (A0, A20, A40) and the three target objects (B, L, C).

using the official NASA TLX app. All data passed the Mauchly's Test of Sphericity. The two-way repeated-measure ANOVA revealed a statistically significant change for this metric across weighting conditions only $(\mathrm{F}(2,28)=13.340, p<0.001)$.

A linear regression was run to understand the effect of $\mathcal{H}_{\mathcal{W}}$ on the final NASA TLX. To assess linearity, a scatterplot of mean $\mathcal{H}_{\mathcal{W}}$ against NASA TLX with superimposed regression line was plotted. Visual inspection of these two plots indicated a linear relationship between the variables. There was homoscedasticity, independence, and normality of the residuals. Average $\mathcal{H}_{\mathcal{W}}$ accounted for $60.7 \%$ of the variation in NASA TLX with adjusted $\mathrm{R}^{2}=60.3 \%$, and it statistically significantly predicted NASA TLX, F $(1,133)=265.123$, $p<0.001$. A Pearson's product-moment correlation showed a statistically significant positive correlation between mean $\mathcal{H}_{\mathcal{W}}$ and NASA TLX, $\mathrm{r}(135)=0.779, p<0.001$.

Fig. 6 shows a representative evolution of both cost functions vs. time for the A0 and A40 weighting schemes.

\section{Discussion AND CONCLUSION}

We presented a haptic shared control for robotic teleoperation that combines human-centered and task-centered cost functions, so as to consider together the need of the human operator as well as the objective of the teleoperation.

First, we devised an innovative approach to estimate the user's muscular comfort during the task, using an inverse kinematic model of the human arm and the popular Rapid Upper Limb Assessment (RULA) tool. This technique can estimate the user's workload $\mathcal{H}_{\mathcal{W}}$ using the pose of the hand registered by the grounded haptic interface, without the need of any additional sensor on the user. Although rather simple and fast to compute, this estimation has shown a good correlation and prediction capability with respect to the NASA TLX results compiled by the user at the end of the task, proving its effectiveness and viability in this scenario. Then, we combined this workload measure with a cost function related to the task at hand or the status of the robotic system. As an example, in our experiments, we considered a cost function $\mathcal{H}_{r}$ indicating the distance from a target position. However, it is important to highlight that the proposed framework supports any other task- or system-related cost function (e.g., trajectory minimizing the energy consumed, displacement, risks of encountering singularities). The one used in this paper has been chosen because representative, effective, and simple to implement. From the combination of $\mathcal{H}_{\mathcal{W}}$ and $\mathcal{H}_{r}$, we then generate a dynamic active constraint guiding the user towards a successful completion of the task along directions maximizing the user comfort.

To prove the effectiveness of the proposed approach, we carried out a robotic telemanipulation experiment enrolling 15 participants. Subjects were asked to pick and place three different sets of objects while receiving three different haptic guidance profiles. The first profile (A0) only considered the task-related cost function $\mathcal{H}_{r}$, guiding the user toward the target position along the shortest path,

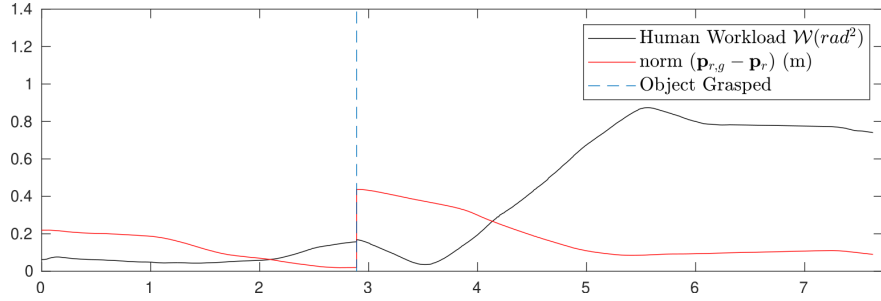

(a) Weighting condition A0 vs. time (s)

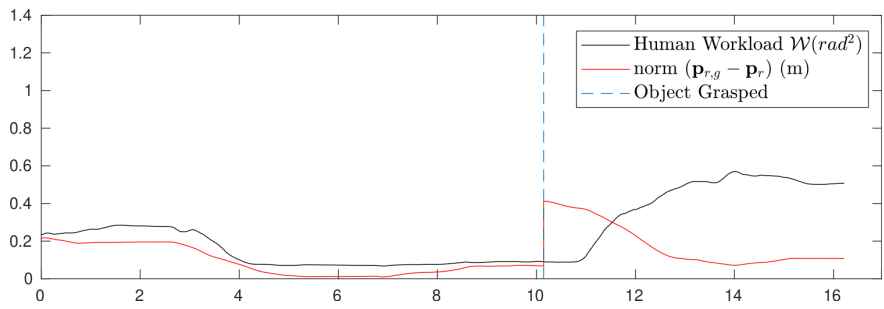

(b) Weighting condition A40 vs. time (s)

Fig. 6. Representative evolution of the human workload metric $\mathcal{W}$ and the norm of the translational error between the current and the desired robot pose, for the A0 and A40 weighing conditions. The user is faster in completing the task using $\mathrm{A} 0$; however, a lower $\mathcal{W}$ is recorded using $\mathrm{A} 40$, especially in the second part of the task (after grasping the object and moving towards the put-down location). The dashed line represents the moment the user grasps the object and the target position changes.

without considering the comfort-related metric $\mathcal{H}_{\mathcal{W}}$. The second guidance profile (A20) started to mix $\mathcal{H}_{\mathcal{W}}$ and $\mathcal{H}_{r}$, guiding the user toward the target position (weight 0.8 ) while also considering the human-centered metric (weight 0.2). The last guidance profile (A40) retains the same mix, but assigning a different importance to $\mathcal{H}_{\mathcal{W}}$ and $\mathcal{H}_{r}$ : weight 0.4 for the former and weight 0.6 for the latter.

We evaluated the performance of the task considering five metrics. Completion time showed a significant degradation when adding our human-centered guidance (A20, A40) vs. standard task-centered guided teleoperation (A0). This result is quite expected, and it is the major drawback of our approach, as the additional guidance inevitably deviates the user from the shortest path. However, this (small) performance degradation is compensated by a significant reduction of the estimated muscular discomfort (mean and max $\mathcal{H}_{\mathcal{W}}$ ) and measured workload (NASA TLX). In fact, while the completion time and placing error degrade by $14 \%$ and $10 \%$, respectively, the NASA TLX value is improved by $30 \%$. It is also important to highlight that we considered a rather short task (approx. 20 minutes in total), and we expect that the effect and usefulness of our approach increase with the duration of the task. Finally, we did not register any noticeable degradation of the performance due to implementing the haptic shared control at the master side. Indeed, where to implement haptic active constraints in robotic teleoperation is still an open question. There are arguments for both. Implementing the active 
constraint at the master side solves any issue related to the stability of the system, but it opens to the risk of badly controlling the slave robot. On the other hand, enforcing the active constraint at the slave side solves any issue related to the commanding of the slave robot, but it exposes to the risks of instabilities.

In the future, we want to explore the possibility of using both (wearable) cutaneous feedback and kinesthetic force feedback to provide our two pieces of information separately, to make it easier for the human operator to differentiate the source of the guidance. Moreover, if we provide the human-centered guidance via a wearable interface, the motion of the user would not be directly affected, possibly leading to smaller errors in carrying out the task. Another option is to provide all feedback information using wearable cutaneous feedback, to inform users about which composite trajectory the system would like them to follow while still leaving them completely free to move wherever they find suitable [35]. We also plan to use other techniques to either directly measure the users' muscle effort (e.g., using Electromyography EMG) or better estimate their pose (e.g., using RGB-D cameras [36], machine-learning approaches, or spatial tracking [37]). Such approaches will solve the uncertainty related to the redundancy of our arm kinematical model. Finally, we plan to carry out further experimental evaluations, considering additional metrics (e.g. jerkiness of the motion, full-body kinematics, workload over a long task and multiple sessions).

\section{REFERENCES}

[1] D. J. Bruemmer, D. A. Few, R. L. Boring, J. L. Marble, M. C. Walton, and C. W. Nielsen, "Shared understanding for collaborative control," IEEE Trans. Systems, Man, and Cybernetics-Part A: Systems and Humans, vol. 35, no. 4, pp. 494-504, 2005.

[2] B. Khademian and K. Hashtrudi-Zaad, "Dual-user teleoperation systems: New multilateral shared control architecture and kinesthetic performance measures," IEEE/ASME Trans. Mechatronics, vol. 17, no. 5, pp. 895906, 2012.

[3] D. Gopinath, S. Jain, and B. D. Argall, "Human-in-the-loop optimization of shared autonomy in assistive robotics," IEEE Robot. Autom. Lett., vol. 2, no. 1, pp. 247-254, 2017.

[4] M. Selvaggio, F. Abi-Farraj, C. Pacchierotti, P. R. Giordano, and B. Siciliano, "Haptic-based shared-control methods for a dual-arm system," IEEE Robot. Autom. Lett., vol. 3, no. 4, pp. 4249-4256, 2018.

[5] V. Duchaine and C. M. Gosselin, "General model of human-robot cooperation using a novel velocity based variable impedance control," in Proc. IEEE World Haptics, 2007, pp. 446-451.

[6] H. Arai, T. Takubo, Y. Hayashibara, and K. Tanie, "Human-robot cooperative manipulation using a virtual nonholonomic constraint," in Proc. IEEE Intl. Conf. Robot. and Autom., vol. 4, 2000, pp. 4063-4069.

[7] R. Rahal, F. Abi-Farraj, P. Giordano, and C. Pacchierotti, "Haptic sharedcontrol methods for robotic cutting under nonholonomic constraints," in Proc. IEEE Intl. Conf. Intelligent Robots and Systems, 2019.

[8] C. Pacchierotti, F. Ongaro, F. Van den Brink, C. Yoon, D. Prattichizzo, D. H. Gracias, and S. Misra, "Steering and control of miniaturized untethered soft magnetic grippers with haptic assistance," IEEE Trans. Automation Science and Engineering, vol. 15, no. 1, pp. 290-306, 2018.

[9] C. Passenberg, A. Glaser, and A. Peer, "Exploring the design space of haptic assistants: the assistance policy module," IEEE Trans. Haptics, vol. 6, no. 4, pp. 440-452, 2013.

[10] D. A. Abbink, M. Mulder, and E. R. Boer, "Haptic shared control: smoothly shifting control authority?" Cognition, Technology \& Work, vol. 14, no. 1, pp. 19-28, 2012

[11] M. Hong and J. W. Rozenblit, "A haptic guidance system for computerassisted surgical training using virtual fixtures," in Proc. IEEE Int. Conf. Systems, Man, and Cybernetics, 2016, pp. 002 230-002 235.

[12] A. M. Ghalamzan, F. Abi-Farraj, P. Robuffo Giordano, and R. Stolkin, "Human-in-the-loop optimisation: mixed initiative grasping for optimally facilitating post-grasp manipulative actions," in Proc. IEEE/RSJ Int Conf. Intelligent Robots and Systems, 2017, pp. 3386-3393.

[13] M. Mulder, D. A. Abbink, and E. R. Boer, "Sharing control with haptics: Seamless driver support from manual to automatic control," Human factors, vol. 54, no. 5, pp. 786-798, 2012.

[14] C. Passenberg, R. Groten, A. Peer, and M. Buss, "Towards real-time haptic assistance adaptation optimizing task performance and human effort," in Proc. IEEE World Haptics Conference, 2011, pp. 155-160.
[15] S. A. Bowyer, B. L. Davies, and F. Rodriguez y Baena, "Active constraints/virtual fixtures: A survey," IEEE Trans. Robotics, vol. 30 , no. 1 , pp. 138-157, 2014

[16] M. Abayazid, C. Pacchierotti, P. Moreira, R. Alterovitz, D. Prattichizzo, and S. Misra, "Experimental evaluation of co-manipulated ultrasoundguided flexible needle steering," International J. Medical Robotics and Computer Assisted Surgery, vol. 12, no. 2, pp. 219-230, 2016.

[17] J. Bimbo, C. Pacchierotti, M. Aggravi, N. Tsagarakis, and D. Prattichizzo, "Teleoperation in cluttered environments using wearable haptic feedback," in IEEE/RSJ Intl. Conf. Intelligent Robots and Systems, 2017, pp. 3401-3408.

[18] L. Meli, C. Pacchierotti, and D. Prattichizzo, "Experimental evaluation of magnified haptic feedback for robot-assisted needle insertion and palpation," International Journal of Medical Robotics and Computer Assisted Surgery, vol. 13, pp. 1809-1809, 2017.

[19] C. Pacchierotti, Cutaneous haptic feedback in robotic teleoperation. Springer, 2015.

[20] F. Chinello, C. Pacchierotti, J. Bimbo, N. G. Tsagarakis, and D. Prattichizzo, "Design and evaluation of a wearable skin stretch device for haptic guidance," IEEE Robot. Autom. Lett., vol. 3, no. 1, pp. 524-531, 2018.

[21] Y. Li, V. Patoglu, and M. K. O'Malley, "Negative efficacy of fixed gain error reducing shared control for training in virtual environments," $A C M$ Trans. Applied Perception, vol. 6, no. 1, pp. 3:1-3:21, 2009.

[22] L. Marchal-Crespo, M. Bannwart, R. Riener, and H. Vallery, "The effect of haptic guidance on learning a hybrid rhythmic-discrete motor task," IEEE Trans. Haptics, vol. 8, no. 2, pp. 222-234, 2015.

[23] B. Busch, G. Maeda, Y. Mollard, M. Demangeat, and M. Lopes, "Postural optimization for an ergonomic human-robot interaction," in Proc. IEEE/RSJ Intl. Conf. Intelligent Robots and Systems, 2017, pp. $2778-2785$.

[24] A. G. Marin, M. S. Shourijeh, P. E. Galibarov, M. Damsgaard, L. Fritzsch, and F. Stulp, "Optimizing contextual ergonomics models in human-robot interaction," in Proc. IEEE/RSJ Intl. Conf. Intelligent Robots and Systems, 2018, pp. 1-9.

[25] L. Chen, L. F. Figueredo, and M. R. Dogar, "Planning for muscular and peripersonal-space comfort during human-robot forceful collaboration," in Proc. IEEE Intl. Conf. Humanoid Robots, 2018, pp. 1-8.

[26] L. Peternel, W. Kim, J. Babi, and A. Ajoudani, "Towards ergonomic control of human-robot co-manipulation and handover," in Proc. IEEE RAS Intl. Conf. Humanoid Robotics, 2017, pp. 55-60.

[27] L. McAtamney and E. N. Corlett, "Rula: a survey method for the investigation of work-related upper limb disorders," Applied ergonomics, vol. 24 , no. 2, pp. 91-99, 1993.

[28] F. Chaumette and S. Hutchinson, "Visual servo control, Part I: Basic approaches," IEEE Robotics and Automation Magazine, vol. 13, no. 4, pp. 82-90, 2006.

[29] M. Shimizu, W.-K. Yoon, and K. Kitagaki, "A practical redundancy resolution for 7 dof redundant manipulators with joint limits," in Proc. IEEE Intl. Conf. Robotics and Automation, 2007, pp. 4510-4516.

[30] H. Seraji, "Configuration control of redundant manipulators: Theory and implementation," IEEE Trans. Robotics and Automation, vol. 5, no. 4, pp. 472-490, 1989.

[31] M. Kang, H. Shin, D. Kim, and S.-E. Yoon, "Torm: Collision-free trajectory optimization of redundant manipulator given an end-effector path," arXiv preprint arXiv:1909.12517, 2019.

[32] F. Abi-Farraj, C. Pacchierotti, O. Arenz, G. Neumann, and P. R. Giordano, "A haptic shared-control architecture for guided multi-target robotic grasping," IEEE Trans. Haptics, 2019.

[33] C. Pacchierotti, D. Prattichizzo, and K. J. Kuchenbecker, "Displaying sensed tactile cues with a fingertip haptic device," IEEE Trans. Haptics, vol. 8, no. 4, pp. 384-396, 2015.

[34] S. G. Hart and L. E. Staveland, "Development of nasa-tlx (task load index): Results of empirical and theoretical research," in Advances in psychology. Elsevier, 1988, vol. 52, pp. 139-183.

[35] C. Pacchierotti, S. Sinclair, M. Solazzi, A. Frisoli, V. Hayward, and D. Prattichizzo, "Wearable haptic systems for the fingertip and the hand: Taxonomy, review, and perspectives," IEEE Trans. Haptics, vol. 10, no. 4, pp. 580-600, 2017.

[36] D. Michel, A. Qammaz, and A. A. Argyros, "Markerless 3d human pose estimation and tracking based on rgbd cameras: an experimental evaluation," in Proc. International Conference on Pervasive Technologies Related to Assistive Environments, 2017, pp. 115-122.

[37] E. Biryukova, A. Roby-Brami, A. Frolov, and M. Mokhtari, "Kinematics of human arm reconstructed from spatial tracking system recordings," Journal of biomechanics, vol. 33, no. 8, pp. 985-995, 2000. 